\title{
Choosing wisely: the message, messenger and method
}

\section{Insights to guide Choosing Wisely activities in Australia, based on a South Australian Clinical Senate exercise}

nternational movements are seeking to identify and reduce the use of health care services that provide little or no benefit, whether through overuse or misuse. England's National Institute for Health and Clinical Excellence commenced a formal policy agenda in this area in $2005 ;{ }^{1}$ however, the first truly physician-driven exercise commenced in 2009 in the United States.

\section{"creating momentum for clinicians to ... take individual and collective responsibility for selecting health practices of limited value"}

The American Board of Internal Medicine Foundation invited professional societies to step forward as "stewards of finite health care resources". ${ }^{2}$ Initially, groups of volunteers from three primary care specialties developed top-five lists. These lists described specialty-specific practice changes that would improve patient outcomes through better treatment choices, reduce risks and, where possible, reduce costs.

In 2012, the program was formally launched as the Choosing Wisely campaign, involving lists from nine specialty societies and including a patient-education component. In 2014, about 50 additional specialty societies joined, and clinicians put forward hundreds of health care practices as being interventions of limited value. ${ }^{2}$

The US Choosing Wisely campaign is unique in how it has framed its message, who has stepped forward as the messenger, and the proposed optimal methods for developing lists of inappropriate health care practices. In this article, we reflect on the relevance of this campaign for Australia.

\section{The message and the messenger}

The lists, entitled Five things physicians and patients should question, set an explicit premise to "spark discussion about the need - or lack thereof - for many frequently ordered tests and treatments". ${ }^{2}$ These lists are freely available to the public via the internet, have been endorsed by consumer organisations and are supported by educational resources for consumers and health care professionals. The main objective

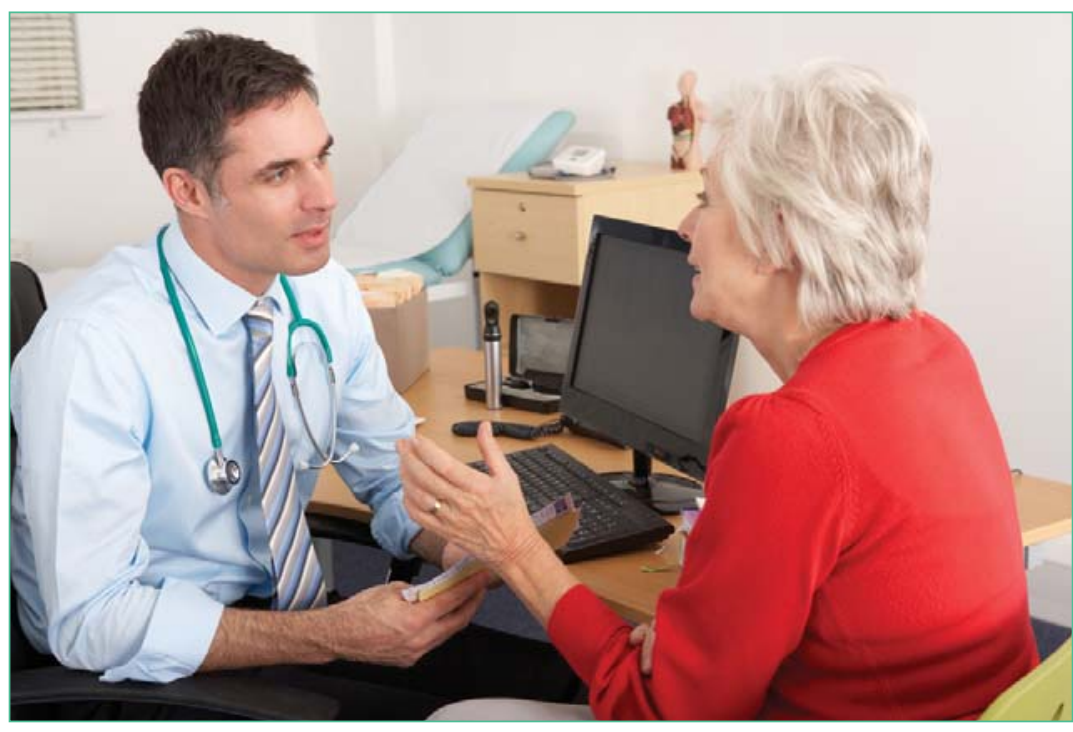

of Choosing Wisely is one of improved safety and quality through a reduction in practices that are, at best, of little to no clinical utility and, in certain situations, harmful. The campaign recognises that the opportunity costs of expending scarce resources on low-value care means they are foregone and cannot be used in other areas where high-value care could have been delivered.

The engagement of about 60 US colleges and professional societies in the program is an acknowledgement by frontline clinicians that in some areas of care, less is more. Clinician leadership by specialty societies and engagement of consumer organisations greatly increase the likelihood of success. The US campaign has, however, been criticised for the variability of methods employed by the specialty groups, and the potential bias this has introduced to the process. ${ }^{3}$
Gerry O'Callaghan MBBCh BAO, FFARCSI, $\mathrm{FCICM}^{1,2}$

Hendrika Meyer

Adam G Elshaug $\mathrm{MPH}, \mathrm{PhD}^{4}$

1 Flinders Medical Centre Adelaide, SA

2 Flinders University, Adelaide, SA.

3 Northern Adelaide Local Health Network, Adelaide, SA.

4 University of Sydney.

gerry.ocallaghan@ health.sa.gov.au

doi: 10.5694/mjal4.00673 MBChB, FACEM, AFCHSM ${ }^{3}$

\section{The method}

International programs such as Choosing Wisely have focused on creating momentum for clinicians to reflect on their work practices and take individual and collective responsibility for selecting health practices of limited value. They aim to change behaviour, rather than rely on policymakers or administrators to achieve similar outcomes.

Although the aims of the US Choosing Wisely campaign have been consistent, the methods employed for list development have not. Some services included on US lists have fallen under scrutiny for varying widely in terms of their potential impact on care and 
spending without a consistent approach in terms of cost, volume or potential for harm. Some societies' lists have been criticised for including low-impact items while excluding high-impact items. ${ }^{4}$ Furthermore, some participating societies have named other specialties' services as low value, avoiding their own.

A recent analysis ${ }^{4}$ showed the most common service types listed by the first 25 Choosing Wisely participants: $29 \%$ of listed items target radiology; $21 \%$, cardiac testing; $21 \%$, medications; $12 \%$, laboratory tests or pathology; and $18 \%$, other. It is clear from the numbers that some interventions have been double counted. It is also feasible that groups (eg, primary care, emergency medicine) are reflecting on the tests they order (eg, radiology, pathology) rather than carry out (and vice versa).

The broadest criticism is that most societies have not detailed the methods by which their lists were created. This has led to one particularly harsh comment,

In some cases, it is clear that the lists were developed without much input from frontline practitioners, using a process that was not transparent and without clear criteria for inclusion ... ${ }^{3}$

At the other end of the spectrum, the method employed by the American College of Emergency Physicians (ACEP) $)^{5}$ has been held up as the gold standard $^{3}$ because it:

- used existing work of the ACEP Cost Effective Care Task Force;

- surveyed the entire ACEP membership and grouped results into domains which were prioritised using serial voting;

- reviewed the literature to establish a solid evidence base that specifically sought to include available cost data; and

- made panellists' disclosures and conflicts of interest publicly available.

The contribution of the ACEP list is as much about methodology (broad college discussion, practitioner surveys, transparency, clinician engagement, modified Delphi technique and solid scientific foundations) as it is about the final recommendations. ${ }^{3}$

A fundamental consideration is to ensure item development is evidence-based. As suggested by existing lists, services that are inappropriate for all patients and indications are rare. Typically, a service has safety and effectiveness profiles that depend on the characteristics of the patient to whom it is provided; a service that is of low value in certain clinical circumstances might be of high value in others. It is precisely for this reason that understanding of the local health care context is critical to identifying practices whose reduction is achievable and will have an impact in terms of quality improvement. ${ }^{6}$

\section{An Australian experience in choosing wisely}

In October 2013, the South Australian Clinical Senate met to consider health care quality improvement. The Senate is a diverse group of practising clinical experts who provide advice to the Minister and Chief Executive of the SA Department of Health and Ageing. The meeting was divided into a morning meeting of emerging clinical leaders and an afternoon session of the full (senior) Clinical Senate. Both groups had the opportunity to hear the case for quality improvement and economic reform of the health system.

As leaders of this clinical consultation group, including an invited content expert (AGE), we asked all senators (junior and senior) to provide, anonymously, their individual list of top-five items in line with the objectives of the Choosing Wisely campaign. Summary results (of the full report ${ }^{7}$ ) are presented in the Box. We

\section{Summary results for the South Australian Clinical Senate Choosing Wisely items}

Health care practice $\quad \begin{gathered}\text { Emerging Clinical Evidence- } \\ \text { leaders senators based list }{ }^{8}\end{gathered}$

Overuse or use not indicated under current guidelines or funding models

Routine inpatient blood tests, electrocardiography and diagnostic imaging for preoperative screening*

CT pulmonary angiography for low-risk pulmonary embolism*

CT of the brain without CNS signs

$\mathrm{CT}$, MRI and $\mathrm{x}$-ray for back pain

C-reactive protein

D-dimer testing

Antibiotics, including prophylactic use*

Proton-pump inhibitors

Statins

Stenting of non-critical coronary arteries

Physiotherapy

Sleep studies

Caesarean section

Bariatric surgery

Tonsillectomy

Prostate-specific antigen testing

Arthroscopy*

Steroid joint injections

Vitamin D testing

Grommets

Cardiac MRI

Surgery for chronic back pain

Robotic surgical techniques

\begin{tabular}{|c|c|c|}
\hline+ & + & + \\
\hline+ & + & - \\
\hline+ & - & + \\
\hline+ & + & + \\
\hline+ & + & + \\
\hline+ & + & - \\
\hline+ & + & - \\
\hline+ & - & - \\
\hline+ & - & - \\
\hline+ & - & + \\
\hline+ & - & + \\
\hline- & + & + \\
\hline- & + & + \\
\hline- & + & - \\
\hline- & + & + \\
\hline- & + & + \\
\hline+ & + & + \\
\hline+ & - & + \\
\hline+ & + & + \\
\hline+ & + & + \\
\hline- & + & - \\
\hline - & + & + \\
\hline- & + & - \\
\hline
\end{tabular}

$\mathrm{CT}$ = computed tomography. $\mathrm{CNS}$ = central nervous system. MRI = magnetic resonance imaging. + Identified by clinicians for inclusion in lists of low-value health care practices. - Not identified by clinicians for inclusion in lists of low-value health care practices. * One of five most commonly identified interventions of potentially limited value. 
provided all participants with a range of preparatory materials, which included an evidence-based list of low-value health care practices. The top-five identified were:

- computed tomography pulmonary angiography for low-risk pulmonary embolism;

- routine inpatient blood tests, electrocardiograms, medical imaging and preoperative screening investigations;

- antibiotics, including prophylactic use;

- arthroscopies in general; and

- duplication of diagnostic testing with change of care teams or between health care contexts.

As might be expected, there was a high level of sensitivity (many selections) and poor specificity (focus). Further, while we often think of bias as

Summary results for the South Australian Clinical Senate Choosing Wisely items (continued)

Health care practice $\quad \begin{gathered}\text { Emerging Clinical Evidence- } \\ \text { leaders senators based list }{ }^{8}\end{gathered}$

Work practice protocols and processes of care

Duplication of diagnostic testing with change of care teams or between contexts*

Excessive outpatient referrals and follow-up

Excessive hospital delivery of care

Duplication of or excessive documentation; process duplication on electronic platforms

Delays in assessment for and discharge to placement

Clinical care delivery by inappropriate health care professional

Innovations in care such as acute medical units, clinical networks, hospital at home

Medical emergency teams

Use of locum and agency health care workers

Time and cost associated with excessive packaging (unpacking, disposing of)

Interventions in the context of limited life expectancy

Cataract surgery

Cardiac surgery

Surgery while on life support

Intensive care

Chemotherapy for advanced malignancy

Implantable cardiac devices

Hip replacements and surgery for fractures

Percutaneous enteric gastrostomy feeding

Faecal occult blood testing

Blood transfusions

Drugs such as statins and vitamin D replacement

na $=$ not applicable. + Identified by clinicians for inclusion in lists of low-value health care practices. - Not identified by clinicians for inclusion in lists of low-value health care practices. $*$ One of the five most commonly identified interventions of potentially limited value. reflecting perverse individual interests (and the protection of these), bias can also merely reflect an awareness of services based on one's own exposure or proximity to their occurrence. Our results showed that relative temporal exposure can affect perceptions of services. This was particularly stark in our findings of differences in choice of top-five items between the emerging and established clinical leadership groups. Junior senators, while offering many purely clinical items, were far more likely to nominate work practices, protocols and processes of care as contributing to areas of low-value care; their senior colleagues focused on therapeutic and diagnostic practices. The role that experience plays is not explored in any Choosing Wisely literature that we have been able to identify, and points to a methodological nuance that any group devising similar initiatives might find valuable.

\section{Conclusions}

Existing lists of low-value services vary in level of cross-representation of items from Choosing Wisely. ${ }^{1,6,8}$ If Australian specialty colleges sought to replicate this initiative, how might they go about maximising list validity while minimising the limitations raised in the US?

We recommend the following steps.

- Consider the methodological principles set out by the ACEP. What constitutes a gold-standard approach is debatable, but it should be transparent and inclusive.

- Consider using existing lists to compile a megalist $\mathrm{t}^{1,2,6,8}$ of items, adding any others nominated by group members.

- Seek explicitly to capture the views of emerging as well as experienced clinicians before working towards prioritisation with (for example) methods employed by the ACEP, and informed as necessary by complementary prioritisation tools. ${ }^{9}$

- As a novel element, establish two top-five lists: one for clinical services and another reflecting administrative, policy and process waste.

- Consider including other health care providers so that all clinical staff are surveyed for their unique, evidence-based perspectives of waste.

Finally, consumer representative organisations must also step forward, as they did in the US, to ensure any campaign complements the role of clinical autonomy while empowering and incorporating patients as partners in choosing wisely.

Competing interests: Adam Elshaug is a member of the Choosing Wisely Australia Advisory Group (NPS MedicineWise)

Provenance: Not commissioned; externally peer reviewed. 
1 Garner S, Littlejohns P. Disinvestment from low value clinical interventions: NICEly done? BMJ 2011; 343: d4519.

2 American Board of Internal Medicine Foundation. Choosing Wisely: an initiative of the ABIM Foundation [website]. 2014. http://www.choosingwisely.org (accessed Jun 2014).

3 Grady D, Redberg RF, Mallon WK. How should top-five lists be developed?: what is the next step? JAMA Intern Med 2014; 174: 498-499.

4 Morden NE, Colla CH, Sequist TD, Rosenthal MB. Choosing Wisely - the politics and economics of labeling low-value services. N Engl J Med 2014; 370: 589-592.

5 Schuur JD, Carney DP, Lyn ET, et al. A top-five list for emergency medicine: a pilot project to improve the value of emergency care. JAMA Intern Med 2014; 174: 509-515.
6 Elshaug AG, McWilliams JM, Landon BE. The value of lowvalue lists. JAMA 2013; 309: 775-776.

7 Government of South Australia, SA Health. SA Clinical Senate report: health technology assessment. October 2013. http://www.sahealth.sa.gov.au/wps/wcm/connect/ public+content/sa+health+internet/resources/sa+clinical+se nate+report+health+technology+assessment+october+2013 (accessed Oct 2014).

8 Elshaug AG, Watt AM, Mundy L, Willis CD. Over 150 potentially low-value health care practices: an Australian study. Med J Aust 2012; 197: 556-560.

9 Elshaug AG, Moss JR, Littlejohns P, et al. Identifying existing health care services that do not provide value for money. Med J Aust 2009; 190: 269-273. 